\title{
Morbilidad de prematuros tardíos: evidencia actual y nuevo enfoque
}

\author{
Álvaro Sepúlveda M. ${ }^{1}$, Stephanie Kobrich S. ${ }^{2}$, Rodolfo Guiñez G. ${ }^{1}$, Jorge Hasbun H. $^{1}$ \\ 1 Unidad de Medicina Materno Fetal, Departamento de Obstetricia y Ginecología, Hospital Clínico, Universidad de Chile. \\ 2 Programa de Posgrado, Departamento de Obstetricia y Ginecología, Hospital Clínico, Universidad de Chile.
}

\section{RESUMEN}

La prematurez es la principal causa de morbimortalidad perinatal, particularmente en los partos prematuros menores a 32 semanas de edad gestacional. Sin embargo, los nacidos entre las 34 y 36 semanas, hasta hace unos años fueron considerados como un grupo de bajo riesgo neonatal. Actualmente se definen como prematuros tardíos y como neonatos de alto riesgo. Se presenta un resumen de la evidencia epidemiológica y de morbimortalidad del prematuro tardío que fundamenta el concepto del mayor riesgo de este grupo tanto en la etapa neonatal como a largo plazo.

PALABRAS CLAVE: Prematuro tardío, distrés respiratorio, morbilidad, mortalidad neonatal, parto prematuro

\section{SUMMARY}

Preterm deliveries are considering the main cause of perinatal morbidity and mortality, mainly those born before 32 weeks of gestational age. However, those born between 34-36 weeks, until recently managed as a low risk newborn, now are defined as late preterm and are considered as high risk children. This review shows a summary of current international evidence related to epidemiology, morbidity and mortality of late preterm, encouraging the concept of high risk of this group not only in neonatal period, but also in long term outcome.

KEY WORDS: Late preterm, respiratory distress, morbidity, neonatal mortality, preterm birth

\section{INTRODUCCIÓN}

Con el requisito de una edad gestacional determinada con seguridad (1), se considera un parto prematuro a todo embarazo que finaliza antes de las 37 semanas. Este grupo ha aumentado aproximadamente un $30 \%$ en los últimos 25 años, alcanzando en algunos países como Estados Unidos tasas cercanas al $12 \%$ (2). Este incremento ocu- rre principalmente en los embarazos entre 32 y 36 semanas, manteniéndose constante la cifra de los prematuros menores a 32 semanas (no más de un $2 \%$ de los nacimientos).

Recientemente se ha definido a los prematuros tardíos (PT) como todo nacimiento producido entre las $34+0$ y las $36+6$ semanas de edad gestacional segura (3), siendo este grupo de prematuros el de mayor incremento en los últimos años. Se ha 
reemplazado el término "near term" $(2,4)$ por el término "prematuro tardío" que identifica en forma más objetiva el riesgo perinatal potencial de este grupo que previamente había sido manejado de modo similar a los partos de término.

Los objetivos del estudio son: revisar la morbilidad e incidencia descritas en las casuísticas internacionales en el grupo definido como PT y determinar la incidencia en la casuística del Hospital Clínico de la Universidad de Chile.

\section{METODOLOGÍA}

Se revisa la literatura internacional en la base de datos Pubmed de todas las publicaciones en el período 2000 al 2011 referente a prematuros tardíos, usando las palabras clave: "late preterm", "outcome", "morbidity", "iatrogenic", "spontaneous", "corticosteroids", "mortality", "cesarean", "preterm birth", "respiratory distress" y "delivery".

Se seleccionan estudios de caso control, cohorte, revisiones sistemáticas y estudios randomizados publicados en inglés y español en revistas indexadas. Se excluyeron del análisis series de casos clínicos sin grupos de comparación, series que no utilizaron el término Prematuro Tardío según definición recomendada por la NICHD (1), obteniéndose finalmente 33 documentos para el análisis final, que fueron revisados por uno de los autores (A.S.).

Se analizan los siguientes datos: tasas de mortalidad neonatal, riesgo de patología neonatal (enterocolitis necrotizante, síndrome de distrés respiratorio, hipoglicemia neonatal, hipomagnesemia neonatal, hiperbilirrubinemia, leucomalacia periventricular, hemorragia intraventricular e hipotermia), admisión a Unidad de Cuidados Intensivos Neonatal, riesgo de cesárea y morbilidad infantil a largo plazo.

\section{EPIDEMIOLOGÍA}

Los PT corresponden a un 9,1\% del total de los nacimientos $(5,6)$ y $71-79 \%$ del total de prematuros (7-11). Las causas de los PT, al igual que todos los prematuros, son principalmente tres: trabajo de parto idiopático, rotura espontánea prematura de membranas (RPM) y por indicación médica o iatrogénicos (PTi), tanto por causas maternas o fetales, considerándose a los dos primeros como prematuros tardíos espontáneos (PTe). Los PTe alcanzan tasas entre un $42-62 \%(5,12-14)$, el resto son por indicación médica por diversas causas, siendo la preeclampsia y la restricción de crecimiento fetal de origen hipóxico las etiologías principales.

En el Hospital Clínico de la Universidad de Chi- le, la tasa de PT entre los años 2001 y 2010 fue de un $7 \%$ del total de nacimientos en embarazos únicos, disminuyendo un $3 \%$ del total de los prematuros entre el primer y segundo quinquenio $(63 \%$ y $60 \%$ respectivamente) (Figura 1 ).

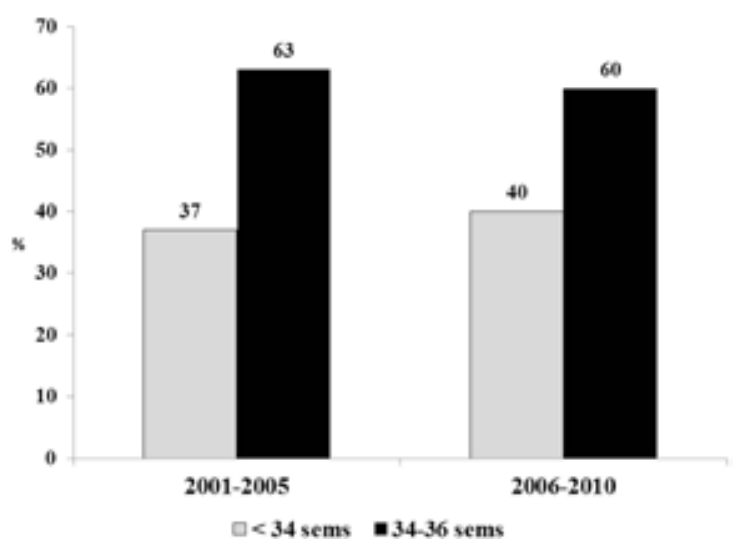

Figura 1. Distribución de partos prematuros según edad gestacional en embarazos únicos (período 2001-2010) Hospital Clínico de la Universidad de Chile.

\section{FISIOPATOLOGÍA}

La mayor incidencia de morbilidad neonatal en PT es por la ausencia de los mecanismos de transición con inadecuada adaptación respiratoria, control glicémico y de temperatura, que pueden extenderse hasta por 24 horas, en comparación con 30 minutos en los nacidos de término (15). Desde el punto de vista respiratorio es necesario el cambio del estado secretor pulmonar, dependiente de los canales de $\mathrm{Cl}^{-}$a un estado absortivo, dependiente de los canales de $\mathrm{Na}+$, lo que permite el clearence adecuado del fluido pulmonar, fenómeno que ocurre frecuentemente a las 39 semanas (16), pero que continúa incluso en el período posnatal por otras vías, como la absorción de fluido pulmonar a través de los vasos linfáticos pulmonares, vasos sanguíneos, a través del mediastino y del espacio pleural (17). Este fenómeno explica la presencia de distrés respiratorio no sólo en los $\mathrm{PT}$, sino que incluso en recién nacidos de término de menos de 39 semanas, aún en presencia de pruebas bioquímicas que confirman madurez pulmonar (18).

\section{MORBIMORTALIDAD NEONATAL}

Publicaciones recientes otorgan a los PTe un 
factor de riesgo independiente de morbilidad neonatal que requiere atención en una Unidad de Cuidados Intensivos Neonatal (UCIN) (12). Sin embargo, los PTi tienen mayor incidencia de sepsis y muerte neonatal que los nacidos de término (14).

Numerosas publicaciones de los últimos años han demostrado un aumento de la morbilidad neonatal de los PT en comparación con los nacidos de término $(3,6,7,9,12,19-21)$, siendo la morbilidad respiratoria la más analizada. El síndrome de distrés respiratorio (SDR) presenta un riesgo entre 11 y 40 veces mayor al compararlo con nacimientos de 39 semanas $(3,6)$, la taquipnea transitoria un Odds Ratio (OR) de 6 a 14 (IC95\%: 5,1-18,4), la neumonía un OR de 3 a 7 (IC95\%: 2,6-11,2) y la falla respiratoria un OR de 6 a 10 (IC95\%: 3,2-16,1) (6). Sin embargo, también destacan otras anormalidades como la hipoglicemia con un OR de 11,4 (IC95\%: 2,54-70,91) (3), la hipotermia, dificultad para la alimentación, necesidad de nutrición parenteral, hiperbilirrubinemia, convulsiones, hemorragia intraventricular, leucomalacia periventricular todas con diferencias significativas. Es importante considerar que en los PT la edad gestacional es el factor predictor más importante para un resultado neonatal adverso, tanto en presencia o ausencia de restricción de crecimiento (22). La morbilidad respiratoria compuesta es de un $22 \%$ a las 34 semanas (OR 24,1; IC95\%: $14,4-40,4), 8,5 \%$ a las 35 semanas (OR 8,9; IC $95 \%: 4,9-16,1$ ) y $3,9 \%$ a las 36 semanas (OR 4,5; IC95\%: 2,6-7,9) (19).

Reforzando este concepto, Bastek y cols (20), analizan 264 nacimientos entre 32 y 39 semanas y demuestra que los resultados neonatales adversos disminuyen $23 \%$ por cada semana que se incrementa desde las 32 semanas en adelante.

Con respecto a la mortalidad, los PT tienen tasas de mortalidad infantil 3 a 6 veces mayores que los nacidos de término $(1,5-7,9 / 1000 \mathrm{nv}$ vs $0,2-$ $2,4 / 1000 \mathrm{nv})(8)(23,24)$, mientras que el riesgo de mortalidad neonatal precoz por cualquier causa es de 4,5 a 5,6 mayor, con tasas de 1,2 a 2,2/1000 nv respectivamente (25).

En 2011, Teune y cols (26), desarrollaron un metaanálisis de 22 estudios de cohorte, abarcando más de 2 millones de PT (período 2000-2010), demostrando que este grupo a pesar de presentar tasas bajas de mortalidad neonatal e infantil, tienen casi 4 veces más riesgo de morir durante el primer año de vida y 3 veces más riesgo de desarrollar parálisis cerebral que los nacidos de término (26).

\section{CESÁREA EN PREMATUROS TARDÍOS}

Aproximadamente un $50-60 \%$ de los PT corres- ponden a partos espontáneos (parto idiopático + rotura prematura espontánea de membranas), finalizando frecuentemente en parto vaginal. A pesar de que la vía vaginal ofrece mayores beneficios en el período neonatal, los PT presentan tasas de cesáreas significativamente mayores que los nacimientos de término. Meloni y cols (4), demostraron tasas de cesárea de $42,9 \%$ en los PT, en comparación con un $22,2 \%$ en los nacidos de término. Al analizar entre los PT iatrogénicos y los espontáneos, Laughon y cols (14), demostraron tasas de cesárea de $56 \%$ y $22 \%$ respectivamente.

Es interesante evaluar la relación de la vía de parto con la morbimortalidad neonatal. Malloy (27), publicó recientemente una comparación de mortalidad neonatal, necesidad de ventilación mecánica e incidencia de membrana hialina en una cohorte de PT, comparando la vía vaginal y cesárea, demostrando que la vía alta se asociaba a un incremento de riesgo de aproximadamente dos veces para los parámetros evaluados, incluso en el análisis separado de las semanas 34,35 y 36 .

\section{DISCUSIÓN}

Los PT es un grupo de riesgo de morbilidad neonatal que ha aumentado en años recientes por el mayor número de interrupciones a esa edad gestacional por indicación médica materna o fetal.

En nuestra institución, a diferencia de lo reportado en la literatura internacional, se ha observado una ligera disminución de las tasas de los PT y un incremento de los prematuros menores de 34 semanas, lo que se explica por la derivación frecuente de embarazos patológicos que requieren manejo de complejidad mayor a edades gestacionales menores.

Aunque comparativamente el mayor costo en recursos médicos está en el grupo de menos de 32 semanas, más vulnerables y de morbilidad más severa a corto y largo plazo, los PT son un grupo de riesgo que pueden beneficiarse con medidas de prevención secundaria o terciaria como el uso de tocolisis y corticoides antenatales en embarazos con riesgo inminente de nacimiento prematuro incluso después de las 34 semanas, en lugar de optar por la evolución espontánea si presenta dinámica uterina dolorosa con membranas intactas, ya que la morbilidad respiratoria ha demostrado ser el evento adverso más frecuentemente identificado, aún cuando todavía no hay evidencia suficiente que respalde esta conducta (28).

Con respecto al uso de corticoides, Bastek y cols (29) analizaron una cohorte de PT con y sin corticoides previo a las 34 semanas, demostrando 
que el grupo tratado tuvo una morbilidad respiratoria significativamente mayor, sin embargo en este resultado paradójico puede haber influido la falta de homogeneidad de la cohorte ya que la rotura prematura de membranas estuvo presente en el grupo tratado y ausente en los controles.

Un estudio reciente, randomizado, controlado con placebo, en 320 pacientes en riesgo de parto entre las 34 y 36 semanas, no logró demostrar reducción de morbilidad respiratoria severa neonatal con el uso de corticoides, incluso luego de ajustar por semana de nacimiento (30).

Aparentemente la falta de efectividad de los corticoides en los PT en estos estudios se debe a la baja incidencia de la patología respiratoria severa en este rango de edad gestacional, por lo que se requiere un número significativamente mayor de pacientes, factible solo con estudios multicéntricos bien diseñados.

También es importante la identificación de aquellos PT iatrogénicos considerados como indicación débil, por ejemplo fetos con restricción de crecimiento pero con perfil biofísico o monitoreo electrónico normal, que en algunas series han llegado a corresponder al 8,8\% del total de los PT (14) y cuya intervención permitiría disminuir las tasas globales de prematurez.

A pesar de que los PT presentan un riesgo elevado de morbilidad neonatal diversa en comparación con embarazos de término, no existe evidencia extensa que realice una comparación entre el subgrupo de PTe con los PTi en donde en el primero aparentemente se observa un feto sano y una madre sin morbilidad severa que justifique una interrupción del embarazo, situación que será motivo de un estudio futuro en nuestra institución. Con respecto a este punto, Shapiro-Mendoza y cols (31), demostraron que en el subgrupo de PT "normales", definidos como aquellos con peso al nacer mayor a $2000 \mathrm{~g}$, de alta antes del cuarto día de nacer y que no requiere mayor atención en el período neonatal inmediato, 4,8\% requirieron rehospitalización (grupo total de 9552 casos), observándose tres muertes en ese subgrupo (31).

Finalmente, es importante considerar el riesgo a largo plazo del PT, recientemente objetivado por MacBird y cols (32), que demostraron un aumento significativo en gastos médicos durante el primer año de vida en 5.188 niños nacidos entre las 34 y 36 semanas, en comparación con 15.303 niños nacidos de término.

Morse y cols (33), demostraron que los PT tienen un riesgo $36 \%$ mayor de retraso del desarrollo hasta los cinco años de vida que los nacidos de término (33), siendo necesario crear conciencia den- tro del ámbito obstétrico de estas observaciones y considerarlas en el manejo de embarazos de estas edades gestacionales, asumiendo conductas con el propósito de disminuir las tasas de PT, ya que son la principal causa del aumento de las tasas de prematuros a nivel global.

\section{CONCLUSIÓN}

El análisis de la evidencia es consistente en demostrar que los $\mathrm{PT}$ representan un grupo de mayor riesgo neonatal, y que mientras no se tengan resultados más definitivos desde el punto de vista de estrategias de prevención, es conveniente asumir las pautas de prevención de los prematuros menores de 34 semanas. La precisión de los riesgos será determinada por estudios multicéntricos, prospectivos, que identifiquen la prematurez tardía por causa (iatrogénico vs espontáneos), por morbilidad específica y por edad gestacional.

\section{REFERENCIAS}

1. Engle W. A recommendation for the definition of "late preterm" (Near-Term) and the birth weight-gestational age classification system. Semin Perinatol 2006;30:27.

2. Davidoff M, Dias Todd, Damus K, Russell R, Bettegowda V, Dolan S, et al. Changes in the gestational age distribution among U.S. singleton births: impact on rates of late preterm birth, 1992 to 2002. Semin Perinatol 2006;30:8-15.

3. Kalyoncu O, Aygun C, Cetinoglu E, Kucukoduk S. Neonatal morbidity and mortality of late-preterm babies. J Matern Fetal Neonatal Med 2010;23:607-12.

4. Meloni A, Antonelli A, Deiana S, Rocca A, Atzei A, Paoletti A, et al. Late preterm: obstetric management. J Matern Fetal Neonatal Med 2010;23(s3):113-5.

5. Reddy U, Wen Ko C, Raju T, Willinger M. Delivery indications at late-preterm gestations and infant mortality rates in the United States. Pediatrics 2009;124:234-40.

6. The Consortium on safe labor. Respiratory morbidity in late preterm births. JAMA 2010;304:419-25.

7. Bastek JA, Sammel MD, Rebele EC, Srinivas S, Elovitz $M$. The effects of a preterm labor episode prior to 34 weeks are evident in late preterm outcomes, despite the administration of betamethasone. Am J Obstet Gynecol 2010;203:140.e1-7.

8. Goldenberg R, Culhane J, lams J, Romero R. Preterm birth 1. Epidemiology and causes of preterm birth. Lancet 2008;371:75-84.

9. Dani C, Corsini I, Piergentili L, Bertini G, Pratesi S, Rubaltelli FF. Neonatal morbidity in late preterm and term infants in the nursery of a tertiary hospital. Acta Paediatrica 2009;98:1841-3.

10. Mclntire D, Leveno KJ. Neonatal mortality and morbidity rates in late preterm births compared with births at term. Obstet Gynecol 2008;111:35-41.

11. Demestre X, Raspall F, Martínez-Nadal S, Vila C, 
Elizari MJ, Sala P. Prematuros tardíos: una población de riesgo infravalorada. An Pediatr (Barc) 2009;71:291-8

12. Melamed N, Klinger G, Tenenbaum K, Herscovici T, Linder $\mathrm{N}$, Hod $\mathrm{M}$, et al. Short-term neonatal outcome in low-risk, spontaneous, singleton, late preterm deliveries. Obstet Gynecol 2009;114:253-60.

13. Holland M, Refuerzo J, Ramin S, Saade G, Blackwell $\mathrm{S}$. Late preterm birth: how often is it avoidable? Am J Obstet Gynecol 2009;201:404.e1-4.

14. Laughon S, Reddy U, Sun L, Zhang J. Precursors for late preterm birth in singleton gestations. Obstet Gynecol 2010;116:1047-55.

15. Laptook A, Jackson G. Cold stress and hypoglycemia in the late preterm ("Near-Term") infant: impact on nursery of admission. Semin Perinatol 2006;30:24-7.

16. Jain $L$, Eaton D. Physiology of fetal lung fluid clearance and the effect of labor. Semin Perinatol 2006;30:34-43.

17. Dudell G, Jain L. Hypoxic respiratory failure in the late preterm infant. Clin Perinatol 2006;33:803-30.

18. Bates E, Rouse D, Mann M, Chapman V, Carlo W, Tita A. Neonatal outcomes after demonstrated fetal lung maturity before 39 weeks of gestation. Obstet Gynecol 2010;116:1288-95.

19. Yoder B, Gordon M, Barth W. Late preterm birth. Does the changing obstetric paradigm alter the epidemiology of respiratory complications? Obstet Gynecol 2008;111:814-22.

20. Bastek J, Sammel M, Paré E, Srinivas S, Posencheg M, Elovitz M. Adverse neonatal outcomes: examining the risks between preterm, late preterm, and term infants. Am J Obstet Gynecol 2008;199:367.e1-8.

21. Saigal S, Doyle L. Preterm Birth 3. An overview of mortality and sequelae of preterm birth from infancy to adulthood. Lancet 2008;371:261-9.

22. Vergani P, Roncaglia N, Ghidini A, Crippa I, Cameroni I, Orsenigo $\mathrm{F}$, et al. Can adverse neonatal outcome be predicted in late preterm or term fetal growth restriction? Ultrasound Obstet Gynecol 2010;36:166-70.

23. Tomashek K, Shapiro-Mendoza C, Davidoff M, Petrini $\mathrm{J}$. Differences in mortality between late-preterm and term singleton infants in the United States, 1995-2002. J Pediatr 2007;151:450-6.

24. Escobar G, Clark R, Greene, J. Short-term outcomes of infants born at 35 and 36 weeks gestation: we need to ask more questions. Semin Perinatol 2006;30:28-33.

25. Kramer M, Demissie K, Yang H. The contribution of mild and moderate preterm birth to infant mortality. JAMA 2000;284:843-9.

26. Teune M, Bakhuizen S, Gyamfi Bannerman C, Opmeer B, van Kaam A, van Wassenaer A, et al. A systematic review of severe morbidity in infants born late preterm. Am J Obstet Gynecol 2011;205:374.e1-9.

27. Malloy M. Impact of cesarean section on intermediate and late preterm births: United States, 2000-2003. BIRTH 2009;36:26-33.

28. Hauth J. Spontaneous preterm labor and premature rupture of membranes at late preterm gestations: to deliver or not to deliver. Semin Perinatol 2006;30:98-102.

29. Bastek J, Sammel M, Rebele E, Srinivas S, Elovitz M. The effects of a preterm labor episode prior to 34 weeks are evident in late preterm outcomes, despite the administration of betamethasone. Am J Obstet Gynecol 2010;203:140.e1-7.

30. Feitosa Porto A, Coutinho I, Barros Correia J, Ramos Amorim M. Effectiveness of antenatal corticosteroids in reducing respiratory disorders in late preterm infants: randomized clinical trial. BMJ 2011;342:d1696. Hallado en: www.ncbi.nlm.nih.gov/pmc/articles/PMC3075234/ pdf/bmj.d1696.pdf

31. Shapiro-Mendoza C, Tomashek K, Kotelchuck M, Barfield W, Weiss J, Evans S. Risk factors for neonatal morbidity and mortality among "healthy", late preterm newborns. Semin Perinatol 2006;30:54-60.

32. MacBird T, Bronstein J, Hall R, Lowery C, Nugent $R$, Mays G. Late preterm infants: birth outcomes and health care utilization in the first year. Pediatrics 2010;126:e311-9.

33. Morse S, Zheng H, Tang Y, Roth J. Early schoolage outcomes of late preterm infants. Pediatrics 2009;123:e622-9. 